\title{
The effect of prolonged monocular occlusion on latent nystagmus in the treatment of amblyopia
}

\author{
H.J. SIMONSZ \\ Universitäts-Augenklinik, Freiburg, BRD; the Laboratory of Medical Physics, University of \\ Amsterdam, The Netherlands; the Netherlands Ophthalmic Research Institute, Amsterdam, \\ The Netherlands
}

Accepted 1 September 1989

Key words: latent nystagmus, amblyopia, occlusion therapy, neural integrator, flocculus

\begin{abstract}
We recorded eye movements in 5 patients with latent nystagmus (LN) before and after 2 days of occlusion of the better eye. The slow-phase speed of the nystagmus (SPS) was in general, before occlusion, lower when the better eye fixated but, after occlusion, lower when the worse eye fixated. However, the sum of SPS during right fixation and SPS during left fixation remained constant. Oscillopsia complaints gradually disappeared during the period of occlusion. These findings indicate that the difference between the SPS during fixation with the right eye and the SPS during fixation with the left eye in LN patients is caused by a compensatory drift that decreases LN during fixation with the better eye but increases LN during fixation with the worse eye. During occlusion, this compensatory drift changes its direction and magnitude slowly over days. Hence, occlusion of the better eye in children with amblyopia and LN should be prescribed only in days per week, not in hours per day.
\end{abstract}

\section{Introduction}

Latent nystagmus (LN) consists of three components: the first component depends on which eye fixates, or intends to fixate $[2,3,8,14,15]$, or on the amount of light [11]. When, for instance, the left eye is covered, the eyes move slowly to the left of the patient. This slow eye movement is interrupted by fast eye movements to the right of the patient. The second component depends on the direction of gaze of the fixating eye $[1,11]$. This gaze-direction component is, in our opinion, Alexander's Law (the magnitude of a nystagmus increases when the patient looks in the direction of the fast phase) applied to latent nystagmus [6]. Alexander's Law is, most likely, an adaptive mechanism that increases the magnitude of the nystagmus when looking in one direction, but decreases the magnitude of the nystagmus when looking in the other direction. It is probably caused by a leaky neural integrator: in normal circumstances the neural integrator keeps the eyes in 
an eccentric position of gaze and counteracts the tendency of the eyes to drift to the primary position. If the neural integrator is not working properly, the eyes drift towards the primary position with an exponentially decreasing speed (a 'centripetal drift' this could be called). This is seen, for instance, in cerebellar lesions. By a similar mechanism, a nystagmus can be compensated for [10]: in one direction of gaze the centripetal drift counteracts the slow phase of the nystagmus. It is clear that the centripetal drift increases the nystagmus in the opposite direction of gaze, but the patient will avoid that direction of gaze. We assume that a similar mechanism is operative in latent nystagmus: the child adducts the fixating eye, and in this position of gaze, the latent nystagmus is counteracted by the centripetal drift caused by a leaky integrator. In one of the patients described below, a recording was made of a latent nystagmus that had extremely few fast nystagmus phases; in this recording, the exponential character of the slow phase, caused by the leaky integrator, was clearly visible.

Finally, LN is often stronger during fixation with the worse eye: this is the third component. Strictly speaking, only the first component is true latent nystagmus, because the second and third components may also occur in other forms of nystagmus.

We investigated this third component by registering eye movements in 5 patients with LN before and after 2 days' occlusion of the better eye. This study was initiated by the controversy among orthoptists about whether children with $\mathrm{LN}$ and amblyopia can be treated by occlusion therapy or not. In short, we found that during prolonged occlusion, the LN that had become manifest by occluding one eye, gradually decreased in intensity. Unexpectedly however, we found that the intensity of the LN during fixation with the occluded eye increased at the same time.

\section{Methods}

The patients were recruited from the strabismus outpatient department. Patients were selected on their ability to cooperate, the magnitude of the LN, and the difference between LN during fixation with the right eye and LN during fixation with the left eye. Eye movements were recorded with an ENG technique (Tönnies DC amplifyer). Horizontal eye movements were registered with two electrodes (Beckman Instruments) placed temporally to each eye on the skin. Vertical eye movements were measured from one eye only, two electrodes being placed above and below one eye. The registration of vertical eye movements in these patients served mainly to register lid twitches and thus avoid artefacts. All electrodes were fixed to the skin at 
least half an hour before registering eye movements to reduce skin resistance. $\mathrm{LN}$ was recorded during binocular, left and right fixation. In addition, $\mathrm{LN}$ was recorded during 20 and 30 deg eccentric gaze, during fixation with one or both eyes, but these recordings are not discussed here. During the registrations, the room was almost dark. Optokinetic stimulation was obtained by projecting a pattern of vertical stripes of light on a curved screen 2 meters in front of the subject. The registrations were recorded on paper (8-channel Siemens $x-t$ recorder, $12 \mathrm{~mm} / \mathrm{s}$ ). After the first recording, the better eye of the patient was patched and the patient was instructed to leave the occlusion in place until the second recording, which took place 2 or 3 days later. All patients gave oral informed consent.

The SPS was determined in each trace of the recorded nystagmus by measurement, by hand, of the angle between slow-phase recording and abscissa, the tangent of this angle being proportional to the SPS. Each time, the angles of 10 randomly-chosen slow phases were measured and averaged. When the slow phase had an exponential waveform (caused by the gazedirection-dependent component, as described above), the tangent to the curve at the middle of the slow phase was taken as slow-phase speed.

\section{Results}

In general, the slow-phase speed (SPS) was, before occlusion, lower when the better eye fixated but, after occlusion, lower then the worse eye fixated. When the difference between SPS during fixation with the right eye and SPS during fixation with the left eye was large before occlusion (in cases of amblyopia), this difference diminished. However, in all cases the sum of SPS during right fixation and SPS during left fixation remained constant. Oscillopsia complains gradually disappeared during the occlusion. Patient 5 was occluded for 3 days. She told us that the oscillopsia had been unbearable the first day (she went to bed), had improved the following day, and had disappeared the third day. She described the oscillopsia as 'those 3-dimensional pictures for children that move when you tilt them'.

\section{Discussion}

The slow-phase speed (SPS) before occlusion was lower when the better eye fixated but, after occlusion; lower when the worse eye fixated. Unexpectedly however, the sum of SPS during right fixation and SPS during left fixation remained constant. 


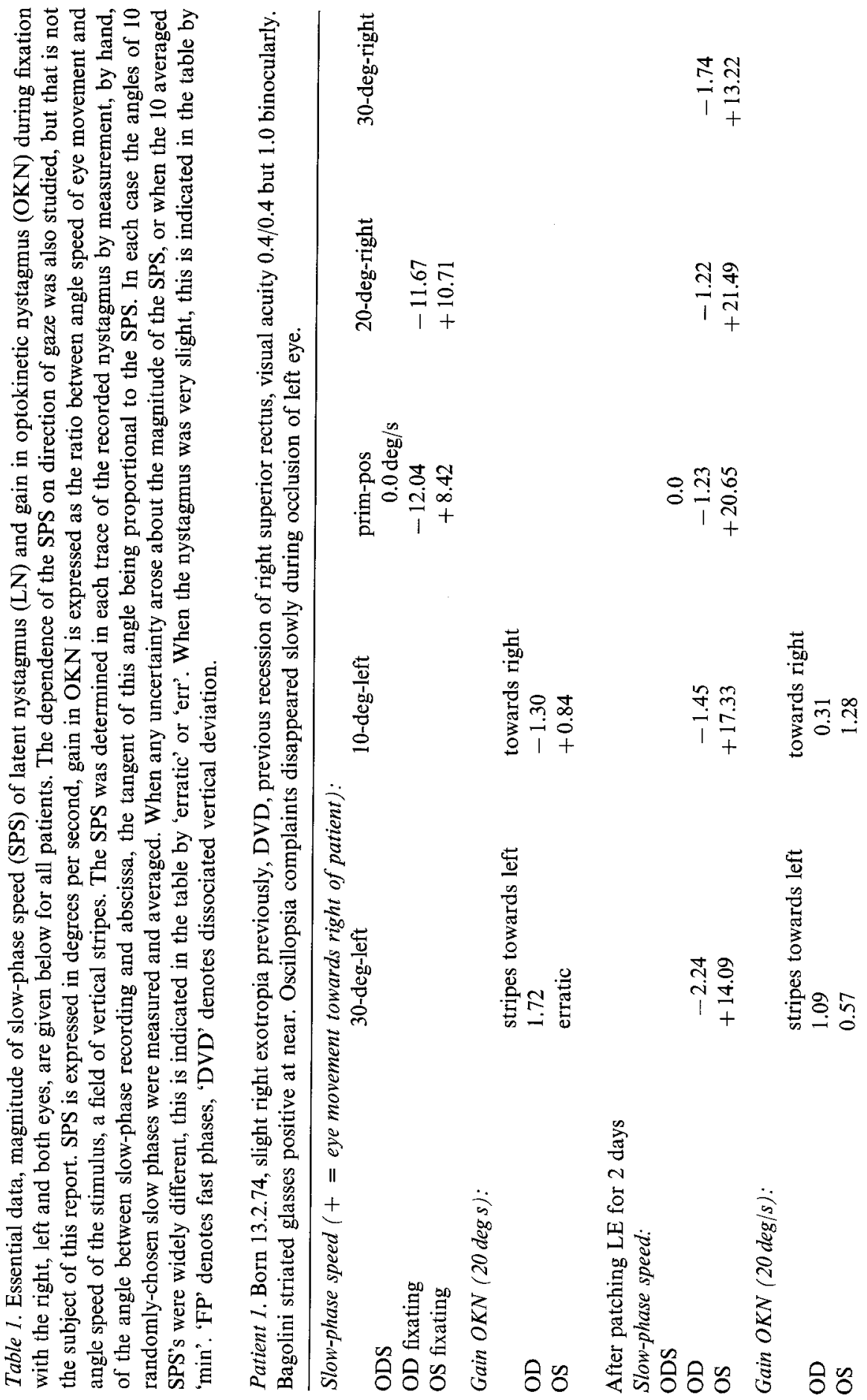




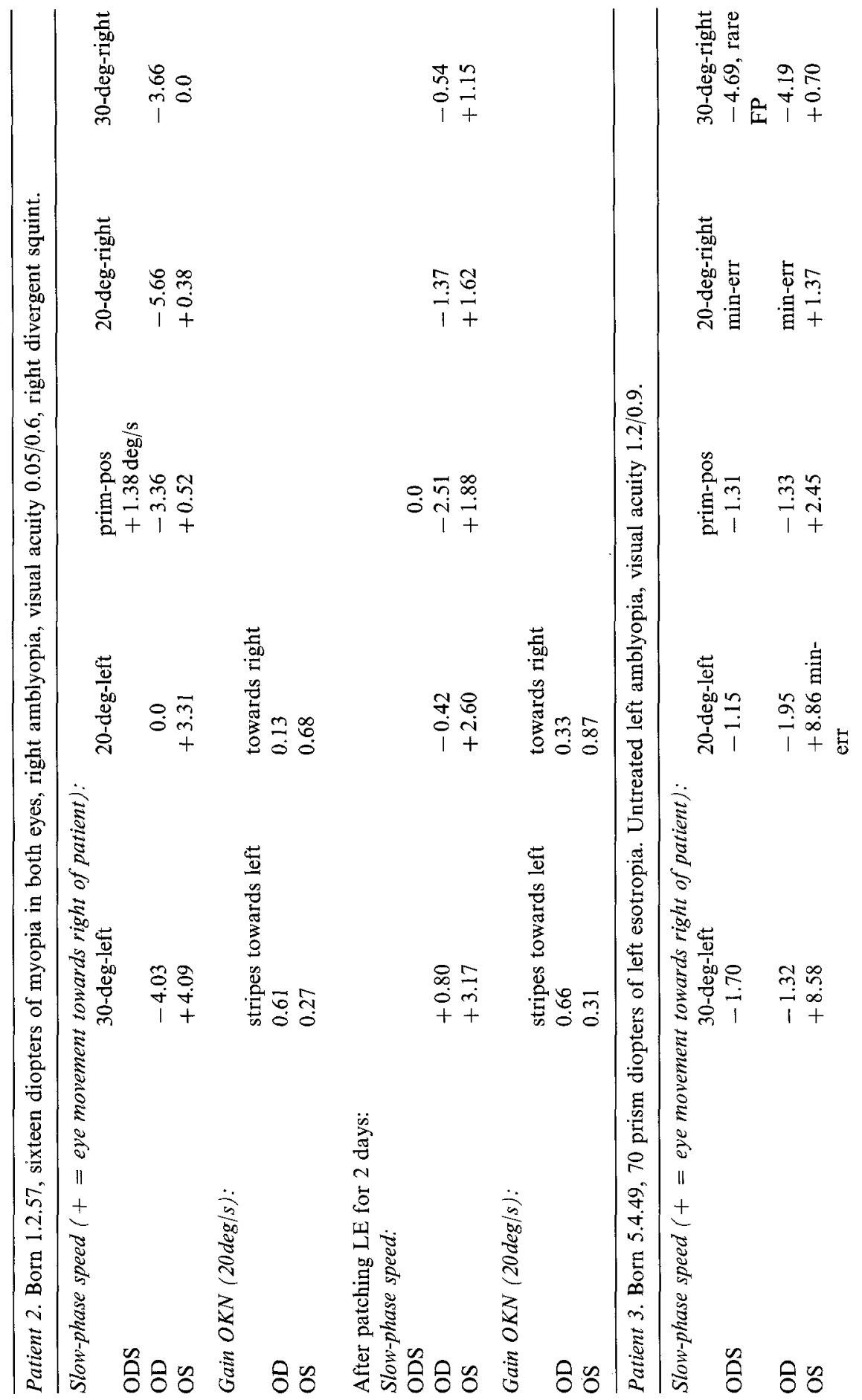




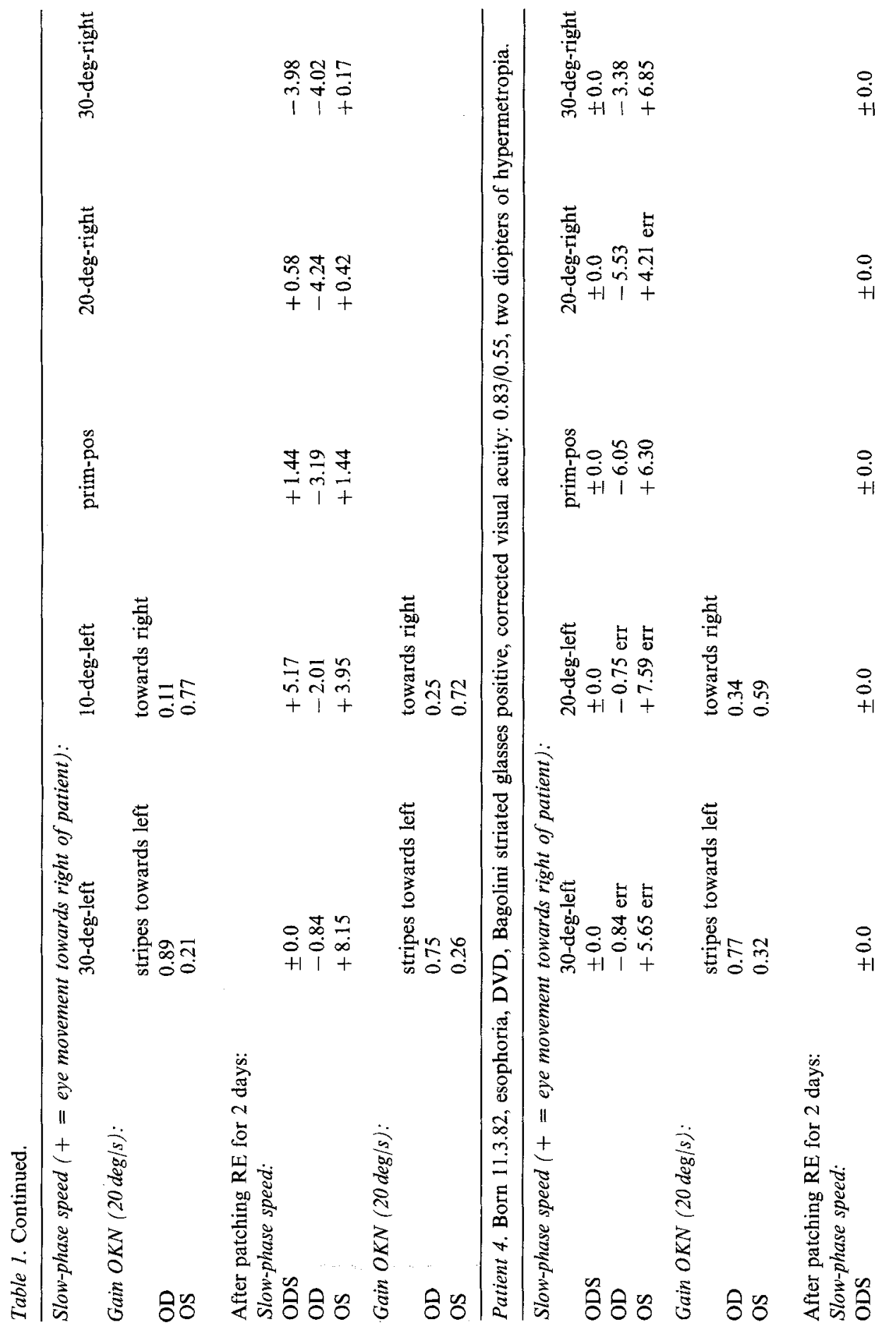




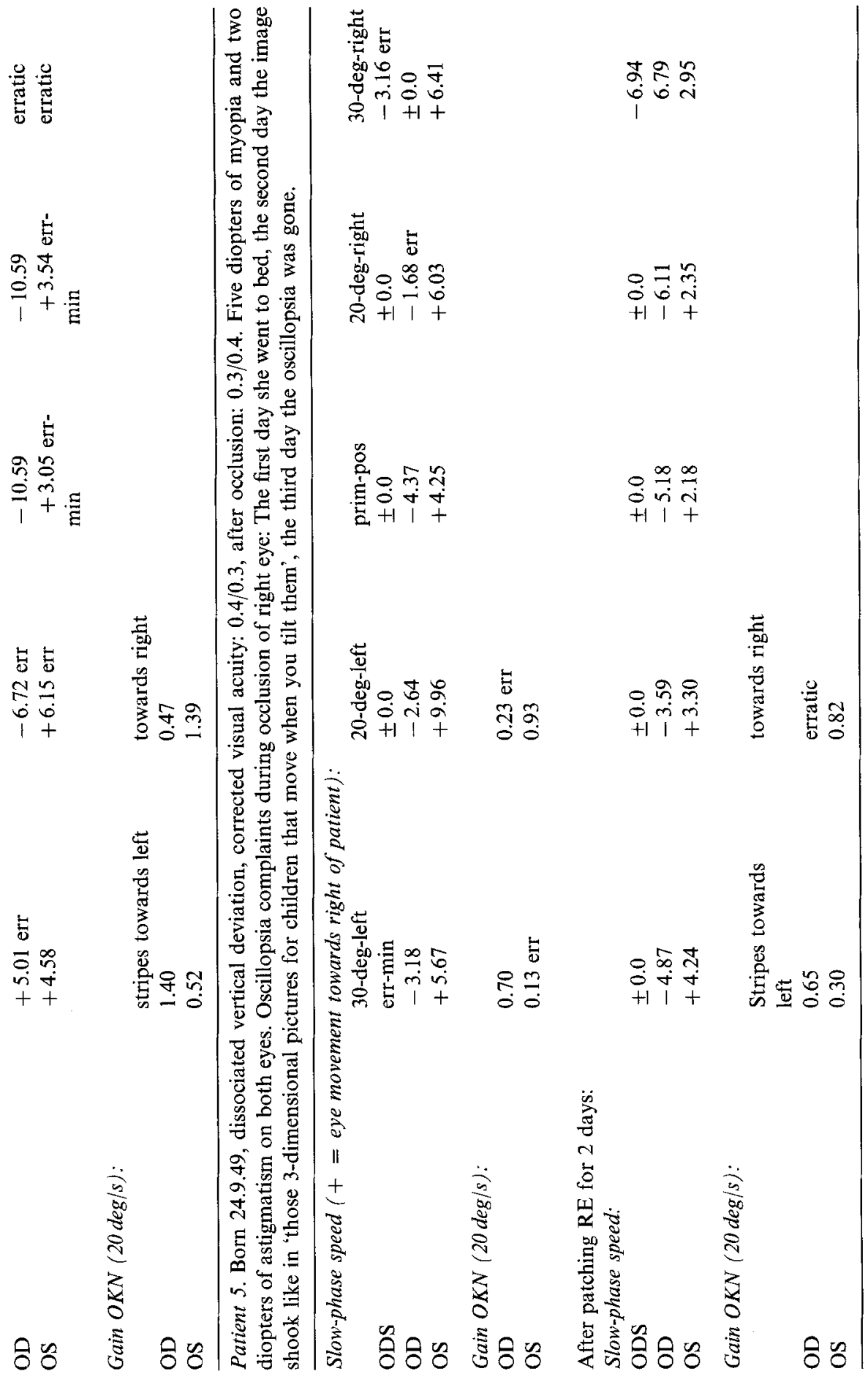


These findings indicate that the difference between the SPS during fixation with the right eye and the SPS during fixation with the left eye in LN patients is caused by a compensatory drift that decreases $\mathrm{LN}$ during fixation with the better eye but increases LN during fixation with the worse eye.

The question remains whether the slow-phase velocity bias was altered by vision or by vestibular circuitry. Alteration of slow-phase velocity bias by vestibular circuitry occurs for instance after unilateral labyrinthectomy. When cats are kept in the dark after unilateral labyrinthectomy, vestibular nystagmus is reduced to approximately one percent after a few days. This reduction is possibly caused by modulation of the VOR by neck afferents. However, when the cats are subsequently kept in a normally lit environment, the remaining one percent also disappears, probably because the flocculus modulates the VOR, employing visual information about the movement of images over the retina (retinal slip). The slow-phase velocity bias becomes manifest if the second labyrinth is also removed. Clinically this is known as Bechterew's nystagmus. Another example of modulation by vestibular circuitry: many first-order vestibular neurons in cats and gerbils, that should in principle register speed of head-rotation only, modulate in response to gravitational changes [4, 9]. Perachio and Correia [9] have suggested that the efferent vestibular system of centrally originating neurons which branch extensively and innervate all receptor organs of the labyrinth, may carry information on static head tilt into the labyrinth. Such a mechanism could also explain the modulation of these neurons during OKN [5].

As an example of modulation by vision, Viirre et al. [13] found monocular adaptation of the saccadic and vestibulo-ocular reflexes after monolateral squint surgery (recession and tenotomy). In these most interesting experiments, monkeys were patched for a week after unilateral recession or tenotomy of a horizontal rectus muscle. The gain in the VOR and the amplitude of the saccades were reduced by between 80 and 90 percent, saccades clearly being more affected when directed towards the operated muscle, and when performed in the field of gaze of the affected muscle. Saccades and VOR of the operated eye were worst on the first postoperative day, recovered slightly on the second postoperative day, but then remained approximately the same from day 2 to day 6 . As soon as the patch was removed, however, saccades and VOR were restored to almost normal within several hours. In other words, vision is the major determinant in this adaptive process. Viirre et al. [12] found earlier that the direction of horizontal eye movements is calibrated by vision and that, in monkeys, horizontal eye movements become skewed after monocular occlusion during one week.

It seems likely that the compensatory drift that we found in patients with $\mathrm{LN}$ is also determined by vision, probably being modulated by the flocculus 
using retinal slip information. This compensatory drift changes its direction and magnitude slowly over days. Hence, occlusion of the better eye in children with amblyopia and LN should be prescribed only in days per week, not in hours per day. Von Noorden et al. [7] have clearly demonstrated that occlusion therapy is not only feasible, but also effective, in cases of amblyopia with latent nystagmus. It is clear that part of the hesitation to treat these children with occlusion therapy has been due to experiences with part-time occlusion: These children should be occluded for days per week, not hours per day.

\section{Acknowledgement}

This work was supported by a fellowship of the Royal Netherlands Academy of Arts and Sciences (Dr Simonsz).

\section{References}

1. Baumeister E. Klinische mededeelingen. III. Invloed van de houding van het hoofd bij de gezichtsscherpte bij nystagmus. Fifteen yearly report concerning nursing and education in the Dutch Clinic for Eye-patients (Scientific suppl.), Donders FC (ed.). Utrecht: Van de Weijer, 1874: 71-3.

2. Dell-Osso LF, Schmidt D, Daroff RB. Latent, manifest latent, and congenital nystagmus. Arch Ophthalmol 1979; 97: 1877.

3. Dell'Osso LF, Abel LA, Daroff RB. Latent/manifest nystagmus reversal using an ocular prosthesis. Invest Ophthalmol 1987; 28: 1873-6.

4. Estes MS, Blanks RHI, Markham CH. Physiological characteristics of first-order canal neurons in the cat. I. Response plane determination and resting discharge characteristics. J Neurophysiol 1978; 38: 1232-49.

5. Henn V, Young LR, Finley C. Vestibular nucleus units in alert monkeys are also influenced by moving visual fields. Brain Res 1974; 71: 144-9.

6. Kommerell G. Ocular motor phenomena in infantile strabismus. In: 'Strabismus and amblyopia', Proc Int Symp Wenner-Gren Center, Stockholm, June 24-26th, 1987. Lennerstrand G, von Noorden GK, Campos EC (eds.). Macmillan Press, 1988: 99-109.

7. von Noorden GK, Avilla C, Sidikaro Y, LaRoche R. Latent nystagmus and strabismic amblyopia. Am J Ophthalmol 1987; 103: 87-9.

8. Ohm J. Der latente Nystagmus im Stockdunkeln. Arch f Augenheilk 1928; 99: 417-37.

9. Percahio AA, Carreia MJ: Responses of semicircular canal and otolith afferents to small static angle head tilts in the gerbil. Brain Research 1983; 280: 287-98.

10. Robinson DA, Zee DS, Hain TC, Holmes A, Rosenberg LF. Alexander's Law: Its behavior and origin in the human vestibulo-ocular reflex. Ann Neurol 1984; 16: 714-22.

11. Roelofs, CO. Nystagmus latens. Arch f Augenheilk 1927; 98: 401-47.

12. Viirre $E$, Cadera $W$, Vilis $T$. The pattern of changes produced in the saccadic system and vestibulo-ocular reflex by visually patching one eye. J of Neurophys 1987; 57: 92-103. 
13. Viirre E, Cadera W, Vilis T. Monocular adaptation of the saccadic system and vestibuloocular reflex. Invest Ophthalmol 1988; 29: 1339-47.

14. van Vliet AGM. Nystagmus latens. Ph.D. thesis, Leiden, 1966.

15. van Vliet AGM. On the central mechanism of latent nystagmus. Acta Ophthalmol 1973; 51: $772-81$.

Address for correspondence: Dr. H.J. Simonsz, oogarts, Netherlands Ophthalmic Research Institute, P.B. 12141, NL 1100 AC, Amsterdam, The Netherlands. 